\title{
QUALIDADE D'ÁGUA E IMPLICAÇÕES NA DINÂMICA SEDIMENTAR NO MÉDIO CURSO DO RIO TUBARÃO, SC
}

\author{
WATER QUALITY AND IMPLICATIONS IN SEDIMENTARY \\ DYNAMICS IN THE MIDDLE COURSE OF RIVER TUBARÃO, \\ SC
}

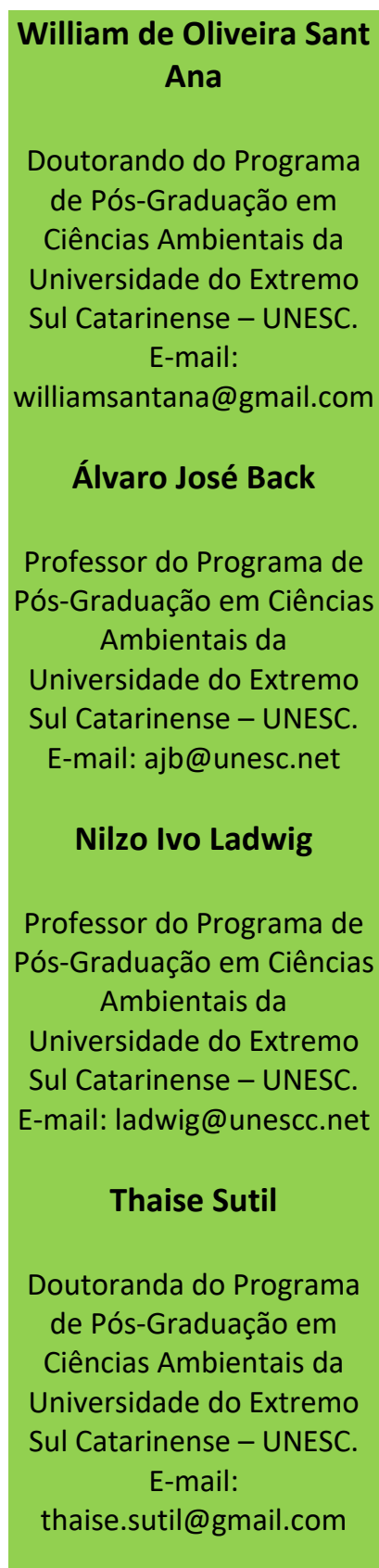

\section{RESUMO}

A Bacia do Rio Tubarão apresenta qualidade ambiental comprometida, com destaque para recebimento de águas oriundas de passivo de mineração. Indicadores de qualidade, a exemplo do $\mathrm{pH}$ reduzido e maior acidez, poderiam acelerar a sedimentação após a confluência dos rios Tubarão (ácido) e Braço do Norte (com muito sedimento). O objetivo deste trabalho consistiu em identificar assinaturas hidroquímicas das águas, antes e após a confluência destes rios, para predizer influência na sedimentação fluvial. Assim, realizou-se amostragem em seis pontos, dois no rio Tubarão, dois no rio Braço do Norte e dois após a confluência de ambos, com nove variáveis químicas: $\mathrm{pH}$, Condutividade Elétrica, $\mathrm{SO}_{4}$, Al, Fe, Mn, Turbidez, Sólidos Sedimentáveis e Oxigênio Dissolvido. Os resultados foram analisados pela Análise de Agrupamento e Análise de Componentes Principais, indicando que o rio Braço do Norte, e as águas após a confluência com o Tubarão, apresentaram forte similaridade. Se é a natureza química da água a promotora das alterações no processo de sedimentação, e se esta é condicionada pelo rio Braço do Norte, como verificado neste trabalho, a hipótese que a acidez e baixo $\mathrm{pH}$ aumentariam as taxas de agradação sedimentar, após o encontro dos rios, foi falseada.

Palavras-chave: Hidroquímica; Sedimentos; Análise Estatística Multivariada.

\section{ABSTRACT}

The Tubarão River Basin has compromised environmental quality, especially the receipt of water from mining liabilities. Quality indicators, such as reduced $\mathrm{pH}$ and higher acidity, could accelerate sedimentation after the confluence of the Tubarão (acid) and Braço do Norte (very sediment) rivers. The objective of this work was to identify hydrochemical signatures of waters, before and after this confluence of these rivers, to predict influence on river sedimentation. Thus, six points were sampled, two in the Tubarão river, two in the Braço do Norte river and two after the confluence of both, listing nine chemical variables: $\mathrm{pH}$, Electrical Conductivity, $\mathrm{SO}^{4}, \mathrm{Al}, \mathrm{Fe}, \mathrm{Mn}$, Turbidity, Sedimentable Solids and Dissolved Oxygen. The results were analyzed by Cluster Analysis and Principal Component Analysis, indicating that the Braço do Norte river, and the waters after the confluence with the Tubarão river, showed strong similarity. If it is the chemical nature of water that promotes changes in the sedimentation process, and if this signature is conditioned 
Revista Tecnologia e Ambiente, v. 25, 2019, Criciúma, Santa Catarina/SC ISSN Eletrônico 23589426 e ISSN Impresso 1413-8131

by the Braço do Norte river, as verified in this paper, the hypothesis that acidity and low $\mathrm{pH}$ would increase sedimentation rates after the meeting of the rivers was falsified.

Keywords: Hydrochemistry; Sediments; Multivariate Statistical Analysis. 
Revista Tecnologia e Ambiente, v. 25, 2019, Criciúma, Santa Catarina/SC ISSN Eletrônico 23589426 e ISSN Impresso 1413-8131

\section{INTRODUÇÃO}

A Bacia Hidrográfica do rio Tubarão está localizada no sul do Estado de Santa Catarina, possui 5.959,97 km², sendo de vertente atlântica e abrangendo 21 municípios (SANTA CATARINA, 2002). A bacia abriga importantes centros regionais, como o município de Tubarão e Laguna, além de crescentes economias, a exemplo de Orleans e Braço do Norte. Seus principais tributários provêm dos contrafortes do Planalto Sul Brasileiro.

A Lei Estadual nº 10.949, de 09 de novembro de 1998, estabelece a divisão de 10 regiões hidrográfica para o Estado de Santa Catarina, visando o planejamento e gerenciamento dos recursos hídricos. De acordo com esta divisão, a bacia está inserida na região hidrográfica denominada Região Hidrográfica 9 - RH9/Sul Catarinense (SANTA CATARINA, 1998). Esta Região Hidrográfica reúne o Complexo Lagunar das lagoas de Imaruí e Mirim e as duas principais bacias hidrográficas: dos rios Tubarão e D’Una, caracterizando-se pelo relevo predominantemente forte ondulado a montanhoso no interior, em contraposição com morfologia plana e suave ondulada junto à Planície Costeira (SIRHESC, 2017).

De acordo com o Sistema de Informações de Recursos Hídricos do Estado de Santa Catarina - SIRHESC (2017), esta bacia hidrográfica é uma das mais comprometidas na sua qualidade ambiental no Estado de Santa Catarina, principalmente quando se considera a carga poluente oriunda da lavra, beneficiamento, transporte e estocagem do rejeito da mineração de carvão, usina termoelétrica, unidades produtoras de coque, fecularias, cerâmicas, e pelo setor agroindustrial (SIRHESC, 2017).

Durante décadas, a principal atividade econômica do sul catarinense foi a extração e beneficiamento do carvão mineral, inclusive nas áreas de cabeceira do rio Tubarão. Análises dos elementos constitutivos deste carvão comprovaram que existe grande proporção de material mineral não aproveitável, no processo de beneficiamento, uma vez que muitos compostos são dispensados e passam a constituir pilhas de rejeitos, que são passivos ambientais. $\mathrm{O} \mathrm{SO}_{4}$ e o $\mathrm{Fe}$, associam-se ao oxigênio nos rios promovendo diminuição do pH e aumento da carga de acidez. Esta diminuição acentuada do potencial de 
Hidrogênio, nas águas do rio, poderia estar acelerando a sedimentação de sólidos suspensos e, por conseguinte, promovendo alterações na dinâmica fluvial.

Os rejeitos da matéria mineral, sem poder calorífico, durante décadas eram despejados nos rios. Nos anos 90, autores já chamavam atenção para o caso:

As cargas de monóxidos e dióxidos de carbono, metano, enxofre, sulfatos, sólidos totais, ferro total, entre outros, lançados diariamente nos rios, juntamente com o inadequado depósito de rejeitos piritosos, preocupam quando relacionados à manutenção de níveis mínimos de qualidade daságuas (Milioli, 1995, p. 34).

A hipótese é de que a interferência antrópica sobre este sistema fluvial altera as condições naturais de fluxo, taxas de agradação e/ou degradação e, em alguns segmentos dos rios, proporcionando a formação de barras, bem como a retirada natural ou antrópica das mesmas, ou mudanças no nível de base (ORFEO, 1996; BROOKFIELD, 2004).

De acordo com Gothe (1988), uma área crítica em que os contaminantes passariam do compartimento água para os sedimentos seria no médio curso do rio Tubarão, com a confluência deste rio com o seu afluente, o rio Braço do Norte (Figura 1), uma vez que esse último, carregado em sólidos suspensos, ao encontrar as águas ácidas do rio Tubarão, favoreceria a precipitação de sedimentos após a confluência destes rios. A poluição aí presente acumular-se-á nos sedimentos finos da porção deltaica do rio Tubarão, o que constituiria num grave problema ambiental, com implicações até de contaminação (CONAMA n. 420/2009).

Os sedimentos são formados por partículas minerais e matéria orgânica que são transportadas ou depositadas nos corpos d'água. Os trabalhos de Santos et al. (2012) já alertavam que cargas de elementos contaminantes nas águas superficiais das bacias hidrográficas podem afetar a qualidade dos sedimentos, isto porque os sedimentos acumulam e redisponibilizam espécies químicas que são, muitas vezes, mais reativas que as formas originais (FÖRSTNER; WITTMAN, 1985).

A problemática de contaminação de metais pesados em sedimentos é tão relevante que a Resolução CONAMA n. 420/2009 estabelece diretrizes para o gerenciamento ambiental de áreas contaminadas por essas substâncias em decorrência de atividades antrópicas, enquanto a CONAMA n. 454/2012 estabelece as diretrizes gerais e os 
procedimentos referenciais para o gerenciamento do material a ser dragado em águas sob jurisdição nacional.

Figura 1 - Contexto da BHRT e detalhe das confluências do rio Tubarão (principal) com seu afluente Braço do Norte

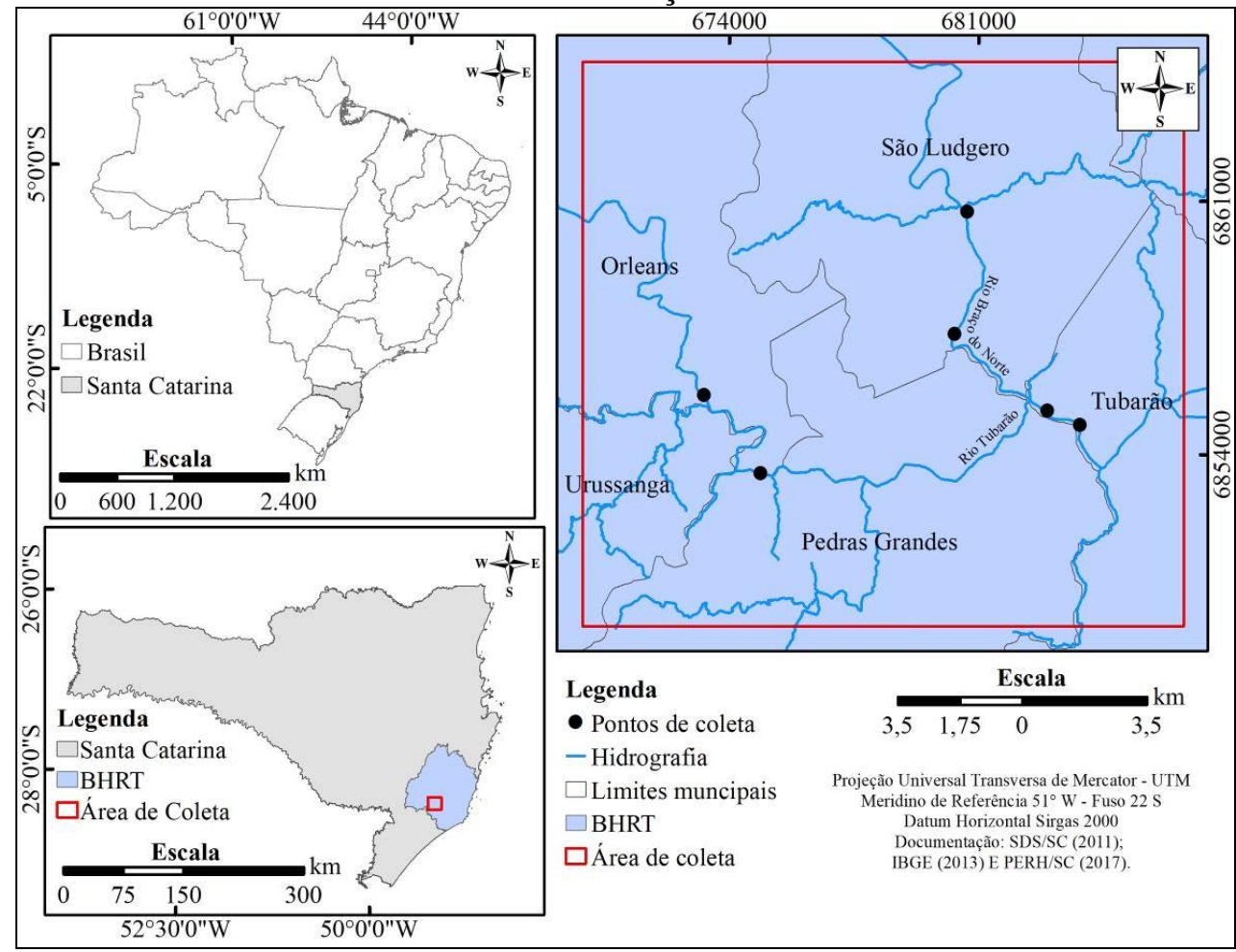

Fonte: Autores (2019)

Diante da problemática levantada, o objetivo deste trabalho consiste em identificar as assinaturas hidroquímicas das águas nas imediações das confluências dos rios Tubarão e Braço do Norte, e suas implicações na sedimentação fluvial. A aplicação e técnicas de estatística multivariada para análise hidroquímica das águas na área de estudo denota originalidade, pois oferece a oportunidade para se averiguar o quanto alguns íons metálicos estão concentrados nas águas. A possível riqueza de componentes químicos dá-se pela implicação antrópica e pela diversidade geológica (WEDEPOHL, 1995), como a Bacia Carbonífera Catarinense e o Embasamento Cristalino, que compõem os terrenos rochosos da área. 
Para atingir o pressuposto, buscar-se-á analisar as assinaturas hidroquímica das águas pelas técnicas de estatística multivariada de análise de agrupamento (Cluster Analysis) e Análise de Componentes Principais (ACP). Ambas apresentam resultados similares e complementares, o que facilita o entendimento da carga iônica e a natureza hidroquímica das águas, com sua possível interferência nos processos sedimentares.

\section{MATERIAIS E MÉTODOS}

As análises laboratoriais de qualidade da água utilizadas neste estudo têm sua origem num levantamento hidroquímico, realizado por técnicos do LAQUA (Laboratório de Análises Químicas e Ambientais) da Associação Beneficente da Indústria Carbonífera de Santa Catarina - SATC, sediado em Criciúma, SC, para analisar a qualidade das águas fluviais na Bacia Carbonífera de Santa Catarina.

No procedimento de aquisição dos dados, optou-se pela estratégia amostral aleatória simples, levando-se em consideração os dois principais formadores da bacia, o Rio Tubarão e o Rio Braço do Norte. Foi efetuada somente uma campanha de coleta durante o mês de abril de 2010, ocasião na qual foram coletadas seis amostras de água. Foram escolhidas nove variáveis químicas, a saber: $\mathrm{pH}$, Condutividade Elétrica, $\mathrm{SO}_{4}, \mathrm{Al}, \mathrm{Fe}, \mathrm{Mn}$, Turbidez (TB), Sólidos Sedimentáveis (SS) e Oxigênio Dissolvido (OD).

Estes dados de levantamento hidroquímico são passíveis de interpretação estatística multivariada. Segundo Neto e Moita (1998), estudos químicos compõem-se de muitas variáveis, algumas redundantes ou menos importantes para algumas análises detalhadas. $\mathrm{O}$ pequeno grupo de variáveis, relevante para cada estudo, deve ser selecionado por critérios mais subjetivos do que objetivos, de acordo com a necessidade de cada pesquisa e experiência do autor.

Varella (2008) aponta que a redução do número de variáveis numa pesquisa, por critérios objetivos, pode ser conseguida através da ACP. Numa plotagem bidimensional euclidiana, é possível se diagnosticar vetores com a devida importância das variáveis para cada caso examinado, bem como, redundâncias. Outra técnica de estatística multivariada, a análise de agrupamento (cluster analysis), reúne casos e variáveis em grupos de 
similaridade que podem ser vistos num dendrograma. Dessa forma, também, pode se selecionar casos e variáveis que se queira detalhar (LANDIM, 2011).

Desta forma, para verificar as semelhanças de alguns casos e as variáveis mais influentes sobre os mesmos, optou-se pelas técnicas de análise de agrupamento e Análise de Componentes Principais (ACP). Como pré-processamento das variáveis, elaborou-se histogramas, com o objetivo de verificar a normalidade. Complementarmente, as variáveis foram transformadas em valores adimensionais (padronizadas) pelos softwares Excel ${ }^{\circledR} \mathrm{e}$ as análises de estatística multivariada e suas representações gráficas obtidas com emprego do software Multi-Variate Statistical Package - MVSP ${ }^{\circledR}$ (LANDIM, 2011).

\section{RESULTADOS}

O recorte espacial da amostragem foi intencionalmente selecionado após duas campanhas de reconhecimento da área de confluência do rio Tubarão com o rio Braço do Norte (Figura 2), inclusive com registros iniciais da dinâmica da geomorfologia fluvial, como a identificação de barras, terraços, planícies de nível de base local, ilhas fluviais e outros.

Figura 2 - Confluência do rio Tubarão (à esquerda) com o rio Braço do Norte (à direita). As setas indicam o sentido de fluxo

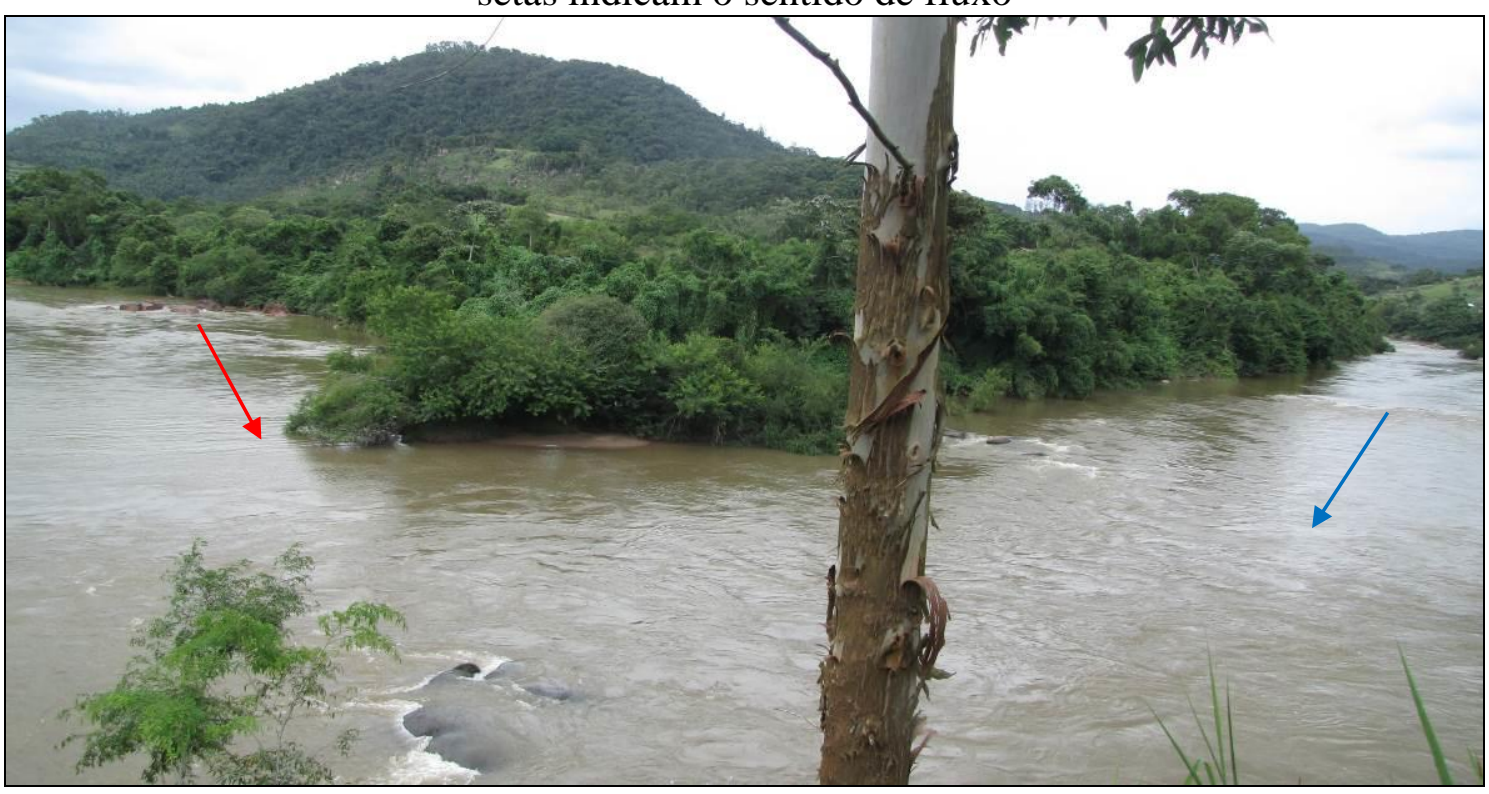

Fonte: Autores (2019). 
Revista Tecnologia e Ambiente, v. 25, 2019, Criciúma, Santa Catarina/SC ISSN Eletrônico 23589426 e ISSN Impresso 1413-8131

Com os dados laboratoriais, obtidos através de análises das águas superficiais, realizou-se a montagem de uma matriz de dados, onde as variáveis independentes foram dispostas nas colunas e as dependentes nas linhas, como segue no Tabela 1. Os pontos em vermelho representam coleta no rio Tubarão (TB), o azul corresponde ao rio Braço do Norte (BN), e o verde após a confluência dos referidos rios (CF) (Figura 3).

Tabela 1 - Resultados das análises químicas dos pontos selecionados em campo

\begin{tabular}{ccccccccccc}
\hline Estação & Grupo & pH & Cond. & Al & Fe & SO $_{\mathbf{4}}$ & Mn & OD & SS & TB \\
\hline PT-01 & TB & 5,80 & 87,00 & 0,69 & 1,87 & 0,00 & 0,00 & 6,30 & 0,00 & 21,00 \\
PT-02 & TB & 4,40 & 131,00 & 1,96 & 1,72 & 0,00 & 0,12 & 5,90 & 0,00 & 18,60 \\
PT-03 & BN & 6,40 & 49,00 & 0,43 & 2,28 & 0,00 & 0,00 & 8,50 & 0,00 & 23,60 \\
PT-04 & BN & 6,70 & 49,00 & 0,43 & 0,89 & 0,00 & 0,00 & 8,40 & 0,00 & 25,00 \\
PT-05 & CF & 6,60 & 56,00 & 0,86 & 0,94 & 0,00 & 0,00 & 8,60 & 0,00 & 25,10 \\
PT-06 & CF & 6,60 & 66,00 & 0,86 & 1,01 & 0,00 & 0,00 & 8,60 & 0,00 & 26,10 \\
\hline
\end{tabular}

Fonte: Autores (2019).

Figura 3 - Mapa de localização dos pontos de coleta

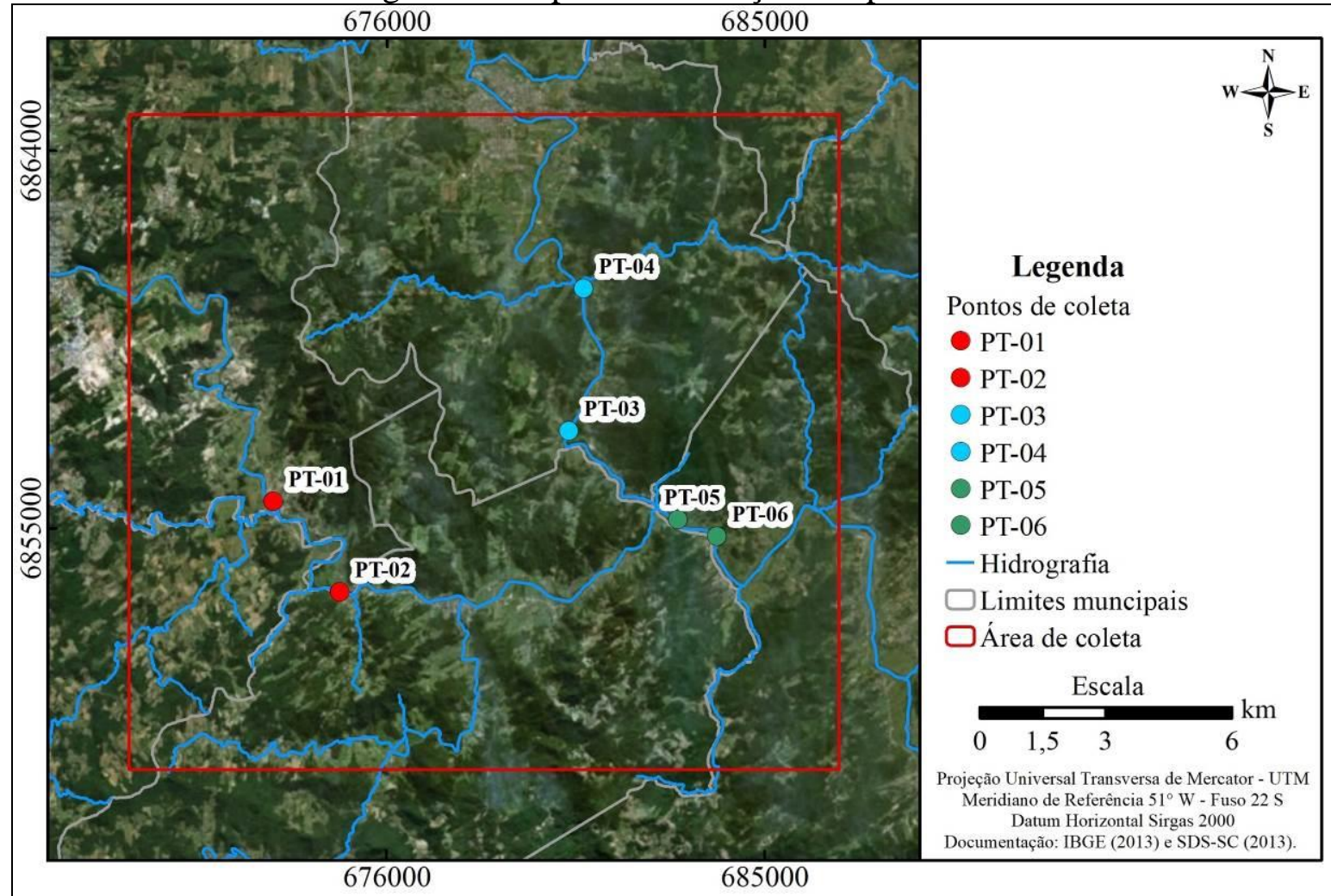

Fonte: Autores (2019). 
A etapa que segue, após montagem da tabela 1 é a transformação dos dados com a padronização e a normalização. Após a padronização e normalização, os dados da Tabela 1 foram importados para o software $M V S P^{\circledR}$. Neste programa efetuou-se uma ACP obtendose, como resultado, a matriz de similaridade (Tabela 2), os eixos principais e a possibilidade de se averiguar os pesos das variáveis conforme distintos eixos (Figura 4). Ressalta-se que estes dados foram inicialmente tratados, sob a técnica de ACP, com todas as variáveis e casos.

Tabela 2 - Resultado da ACP no software MVSP para os dados da Tabela 1. Detalhe para os dados normalizados $(\log 10)$ e padronizados, bem como pesos dos eixos e de variáveis

nos mesmos.

\begin{tabular}{|c|c|c|c|c|c|c|c|c|c|}
\hline \multicolumn{10}{|c|}{ PRINCIPAL COMPONENTS ANALYSIS } \\
\hline \multicolumn{10}{|c|}{ Analysis begun: segunda-feira, 26 de abril de 2010 16:48:05 } \\
\hline \multicolumn{10}{|c|}{ Analysing 9 variables $\times 6$ cases } \\
\hline \multicolumn{10}{|c|}{2 variables and 0 cases have been dropped from original data } \\
\hline \multicolumn{10}{|c|}{ Data $\log (10)$ transformed } \\
\hline \multicolumn{10}{|c|}{ Tolerance of eigenanalysis set at $1 \mathrm{E}-007$} \\
\hline \multicolumn{10}{|l|}{ Data standardized } \\
\hline \multicolumn{10}{|l|}{ Similarity matrix } \\
\hline $\mathrm{pH}$ & Cond. & $\mathrm{Al}$ & $\mathrm{Fe}$ & SO4 & $\mathrm{Mn}$ & OD & SS & TB & \\
\hline $\mathrm{pH}$ & & & & & & & & & \\
\hline$-0,929$ & 1 & & & & & & & & \\
\hline$-0,854$ & 0,878 & 1 & & & & & & & \\
\hline$-0,464$ & 0,327 & 0,067 & 1 & & & & & & \\
\hline SO4 & 0 & 0 & 0 & 0 & & & & & \\
\hline$-0,944$ & 0,829 & 0,897 & 0,264 & 0 & 1 & & & & \\
\hline 0,895 & $-0,919$ & $-0,66$ & $-0,497$ & 0 & $-0,717$ & 1 & & & \\
\hline SS & 0 & 0 & 0 & 0 & 0 & 0 & 0 & & \\
\hline 0,95 & $-0,87$ & $-0,677$ & $-0,624$ & 0 & $-0,811$ & 0,955 & 0 & 1 & \\
\hline $\mathrm{pH}$ & Cond. & $\mathrm{Al}$ & $\mathrm{Fe}$ & $\mathrm{SO} 4$ & $\mathrm{Mn}$ & OD & SS & TB & \\
\hline \multicolumn{10}{|l|}{ Eigenvalues } \\
\hline & Axis 1 & Axis 2 & Axis 3 & Axis 4 & Axis 5 & Axis 6 & Axis 7 & Axis 8 & Axis 9 \\
\hline Eigenvalues & 5,462 & 1,088 & 0,302 & 0,13 & 0,017 & 0 & 0 & 0 & 0 \\
\hline Percentage & 78,035 & 15,541 & 4,318 & 1,861 & 0,245 & 0 & 0 & 0 & 0 \\
\hline Cum. Percentage & 78,035 & 93,576 & 97,894 & 99,755 & 100 & 100 & 100 & 100 & 100 \\
\hline \multicolumn{10}{|l|}{ PCA variable loadings } \\
\hline & Axis 1 & Axis 2 & Axis 3 & Axis 4 & Axis 5 & Axis 6 & Axis 7 & Axis 8 & Axis 9 \\
\hline $\mathrm{pH}$ & $-0,425$ & 0,021 & $-0,143$ & $-0,181$ & $-0,097$ & $-0,353$ & 0,795 & 0 & 0 \\
\hline Cond. & 0,409 & $-0,128$ & $-0,339$ & $-0,464$ & 0,527 & 0,361 & 0,281 & 0 & 0 \\
\hline $\mathrm{Al}$ & 0,364 & $-0,446$ & 0,244 & $-0,527$ & $-0,532$ & $-0,219$ & $-0,031$ & 0 & 0 \\
\hline $\mathrm{Fe}$ & 0,204 & 0,808 & 0,387 & $-0,362$ & 0,058 & $-0,145$ & 0,018 & 0 & 0 \\
\hline $\mathrm{SO} 4$ & 0 & 0 & 0 & 0 & 0 & 0 & 0 & 0 & 1 \\
\hline $\mathrm{Mn}$ & 0,39 & $-0,241$ & 0,517 & 0,431 & 0,382 & $-0,295$ & 0,322 & 0 & 0 \\
\hline OD & $-0,397$ & $-0,151$ & 0,611 & $-0,153$ & 0,016 & 0,633 & 0,15 & 0 & 0 \\
\hline SS & 0 & 0 & 0 & 0 & 0 & 0 & 0 & 1 & 0 \\
\hline
\end{tabular}


Revista Tecnologia e Ambiente, v. 25, 2019, Criciúma, Santa Catarina/SC ISSN Eletrônico 2358-

9426 e ISSN Impresso 1413-8131

\begin{tabular}{|c|c|c|c|c|c|c|c|c|c|c|}
\hline \multicolumn{2}{|l|}{ ТВ } & $-0,41$ & $-0,226$ & 0,121 & $-0,366$ & 0,53 & $-0,434$ & $-0,403$ & 0 & 0 \\
\hline \multicolumn{11}{|c|}{ PCA case scores } \\
\hline & Groups & Axis 1 & Axis 2 & Axis 3 & Axis 4 & Axis 5 & Axis 6 & Axis 7 & Axis 8 & Axis 9 \\
\hline PT-01 & ТВ & 0,446 & 0,482 & $-0,408$ & $-0,058$ & $-0,01$ & 0 & 0 & 0 & 0 \\
\hline PT-02 & TB & 1,943 & $-0,239$ & 0,143 & 0,051 & 0,006 & 0 & 0 & 0 & 0 \\
\hline PT-03 & $\mathrm{BN}$ & $-0,463$ & 0,694 & 0,325 & $-0,009$ & 0,002 & 0 & 0 & 0 & 0 \\
\hline PT-04 & $\mathrm{BN}$ & $-0,801$ & $-0,186$ & $-0,092$ & 0,277 & 0,034 & 0 & 0 & 0 & 0 \\
\hline PT-05 & $\mathrm{CF}$ & $-0,58$ & $-0,375$ & 0,027 & $-0,05$ & $-0,103$ & 0 & 0 & 0 & 0 \\
\hline PT-06 & $\mathrm{CF}$ & $-0,544$ & $-0,376$ & 0,005 & $-0,212$ & 0,072 & 0 & 0 & 0 & 0 \\
\hline
\end{tabular}

Fonte: Autores (2019).

As matrizes obtidas pela ACP demonstram a importância ou redundância de variáveis sobre os casos, contudo, é através dos gráficos euclidianos bidimensionais, obtidos como complemento de cada análise detalhada, que fica fácil a interpretação dos resultados numéricos. As Figuras 4 e 5 apresentam dois destes gráficos, que cruzam, respectivamente, os eixos 1 e 2 / 2 e 1 obtidos nos resultados da ACP, conforme Tabela 2. Desta forma as possíveis relações entre variáveis e casos puderam ser percebidas conforme a proximidade dos pontos, que aparecem plotados nestas figuras, bem como a distância dos vetores das variáveis.

Figura 4 - Cruzamento dos eixos 1 e 2. Na legenda os TB são pontos do rio Tubarão, BN pontos no rio Braço do Norte e CF- pontos após a Confluência.

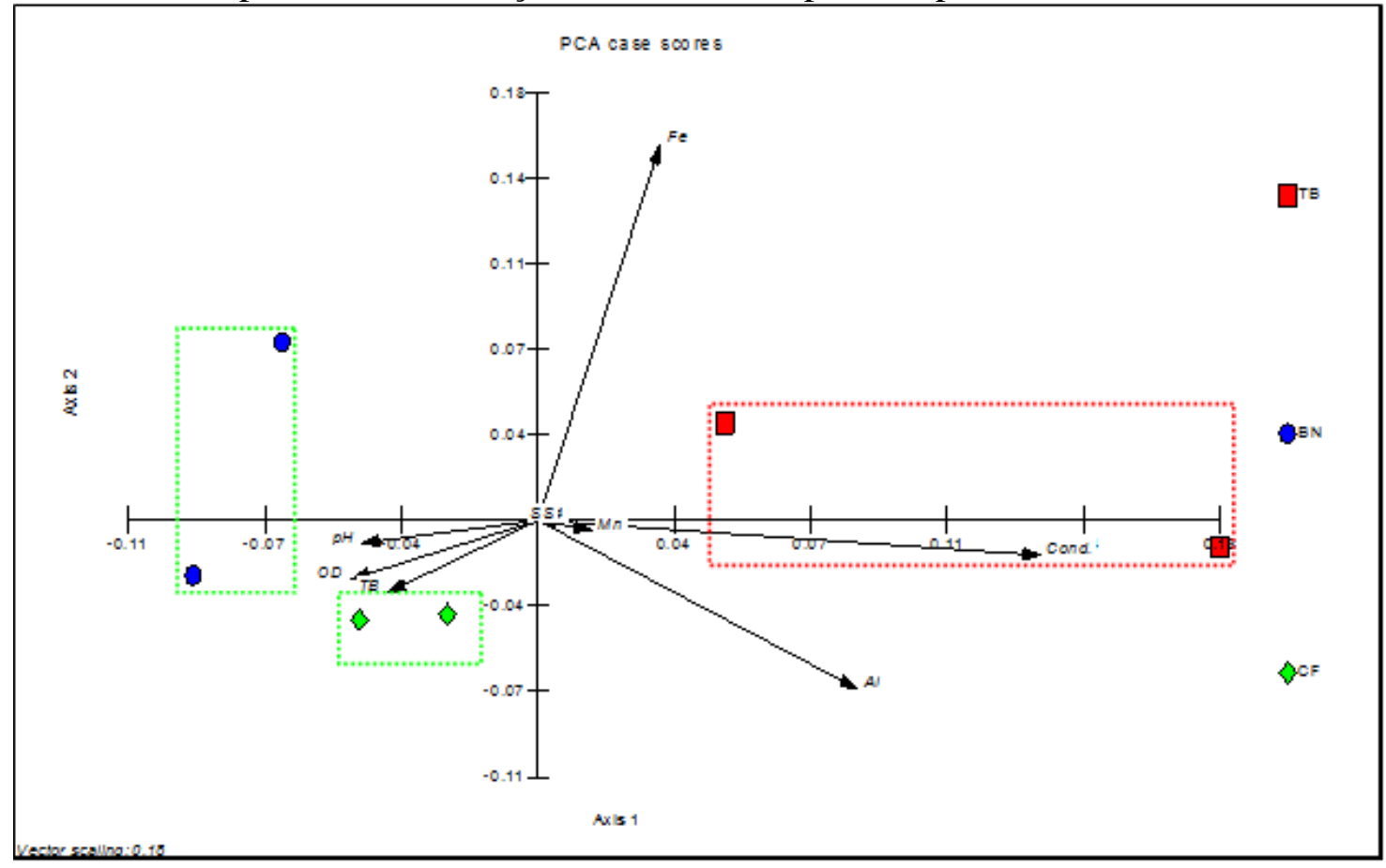

Fonte: Autores (2019). 
Na Figura 4 ficou evidente que que os dois pontos de análises de águas do rio Tubarão apresentam maiores discrepâncias entre si, todavia, estão homogeneamente concentradas à direita da figura, sob influência redundante de vetores de variáveis que representam íons metálicos, como o $\mathrm{Fe}, \mathrm{Mn}$ e $\mathrm{Al}$, além da condutividade (descritor). Estas características hidroquímicas reafirmam que o rio Tubarão concentra águas de despejos industriais, inclusive de áreas que drenam passivo ambiental de mineração.

As águas do rio Braço do Norte e as águas após a confluência apresentaram maior proximidade na Figura 4, denotando, que após a confluência das águas, as características hidroquímicas do rio Braço do Norte sobressaem, diluindo a concentração de íons metálicos. Da mesma forma, o pH, os Sólidos Sedimentáveis e o Oxigênio Dissolvido, das águas após a confluência, são determinados preponderantemente pelas características hidroquímicas do rio Braço do Norte. Para efeito de comparação com a Figura 4, plotou-se a Figura 5, que segue abaixo:

Figura 5 - Cruzamento dos eixos 2 e 1. Na legenda os TB são pontos do rio Tubarão, BN pontos no rio Braço do Norte e CF- pontos após a Confluência.

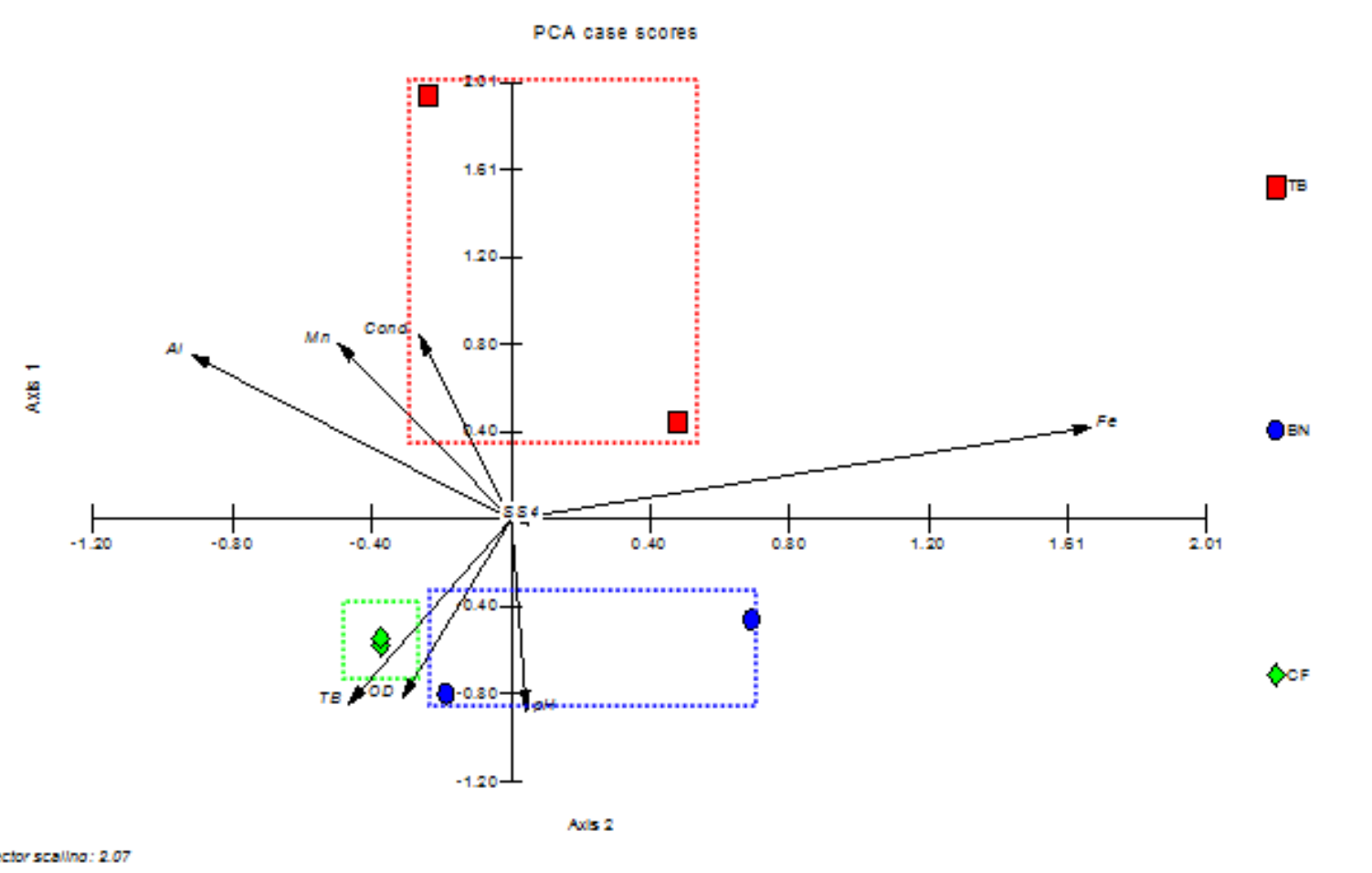

Fonte: Autores (2019). 
A Figura 5 foi complementar a Figura 4 e serviu para ratificar as conclusões sobre o mesmo. Ainda fica perceptível o arranjo diferenciado dos pontos do rio Tubarão em relação aos casos com diferentes pontos de coleta de água, possuindo estas águas maior carga metálica.

As Figuras 6 e 7 demonstram um dendrograma "modo q" (casos) com a similaridade das dos diferentes pontos de coleta. Esta análise foi complementar ao diagrama bidimensional euclidiano, feito a partir da técnica de ACP, e nela averigua-se os mesmos grupos: em vermelho o grupo 1, com as águas do rio Tubarão, em azul o grupo 2, com águas do rio Braço do Norte e em verde o grupo 3, com a mistura de águas após a confluência dos referidos rios. Uma linha de corte (magenta) foi usada aleatoriamente como referência para a identificação dos grupos e para ratificar as distâncias entres os pontos e grupos existentes. Esta linha pode-se deslocar tanto para direita como para esquerda, conforme a análise que se deseja realizar.

Figura 6 - Dendrograma resultante da análise de agrupamento para os pontos de coleta. As cores e os grupos propostos no diagrama euclidiano são coincidentes

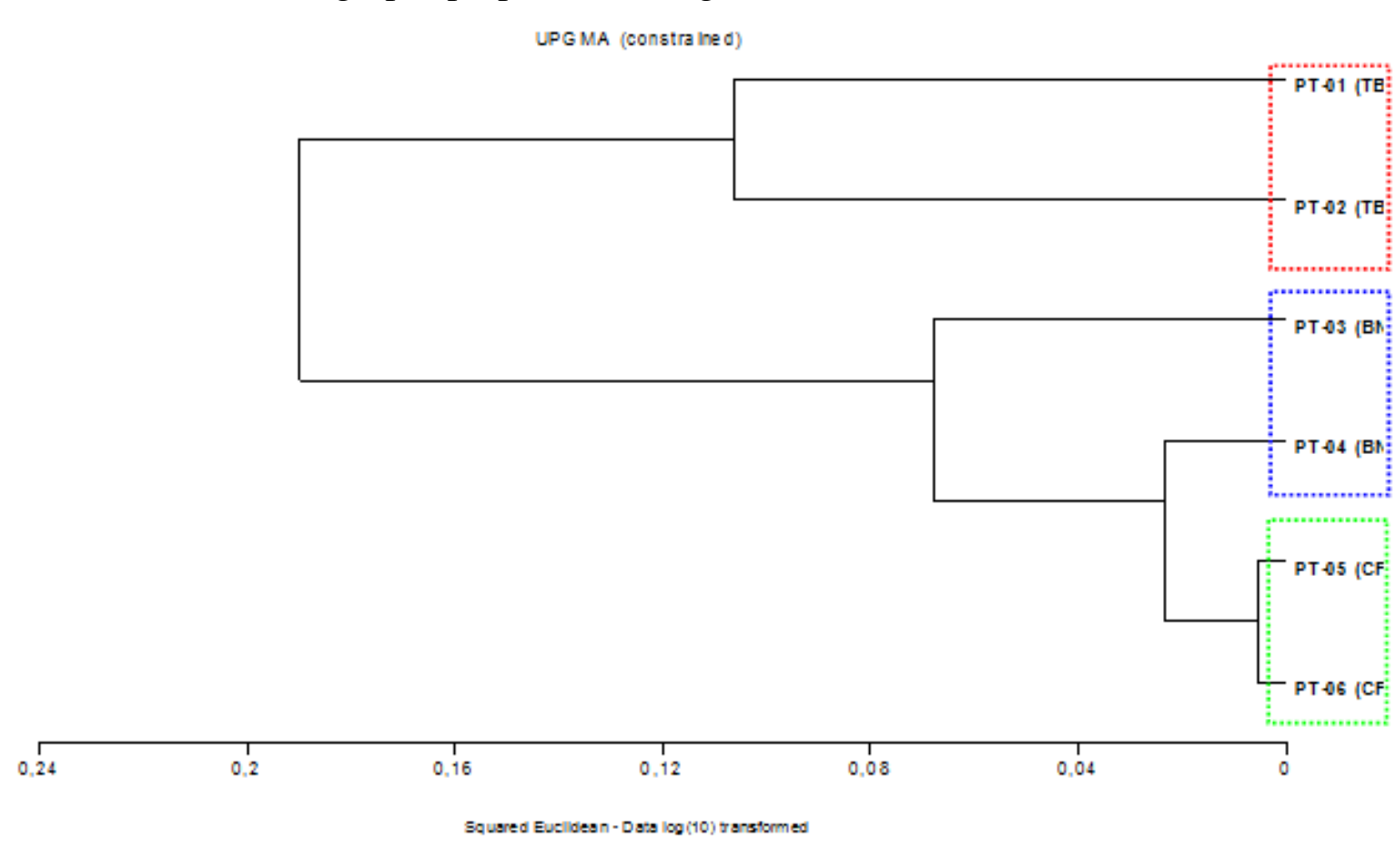

Fonte: Autores (2019). 
De acordo com a formação dos grupos, pode-se observar a distância entre os pontos de um mesmo grupo (caso do rio Tubarão), e a proximidade dos pontos do grupo azul e verde, ressaltando que as águas do rio Braço do Norte são perceptivelmente semelhantes às águas após a confluência entre os rios.

Um segundo dendrograma (Figura 7) foi elaborado com uma técnica diferente, para validar os resultados e, consequentemente, foi comprovada a proximidade das águas do rio Braço do Norte com as da confluência. Na Figura 7 foi mantida a mesma escala para linha de corte e as interpretações complementaram as realizadas para a Figura 6.

Figura 7 - Dendrograma resultante da análise de agrupamento para os pontos de coleta. As cores e os grupos propostos no diagrama euclidiano são coincidentes

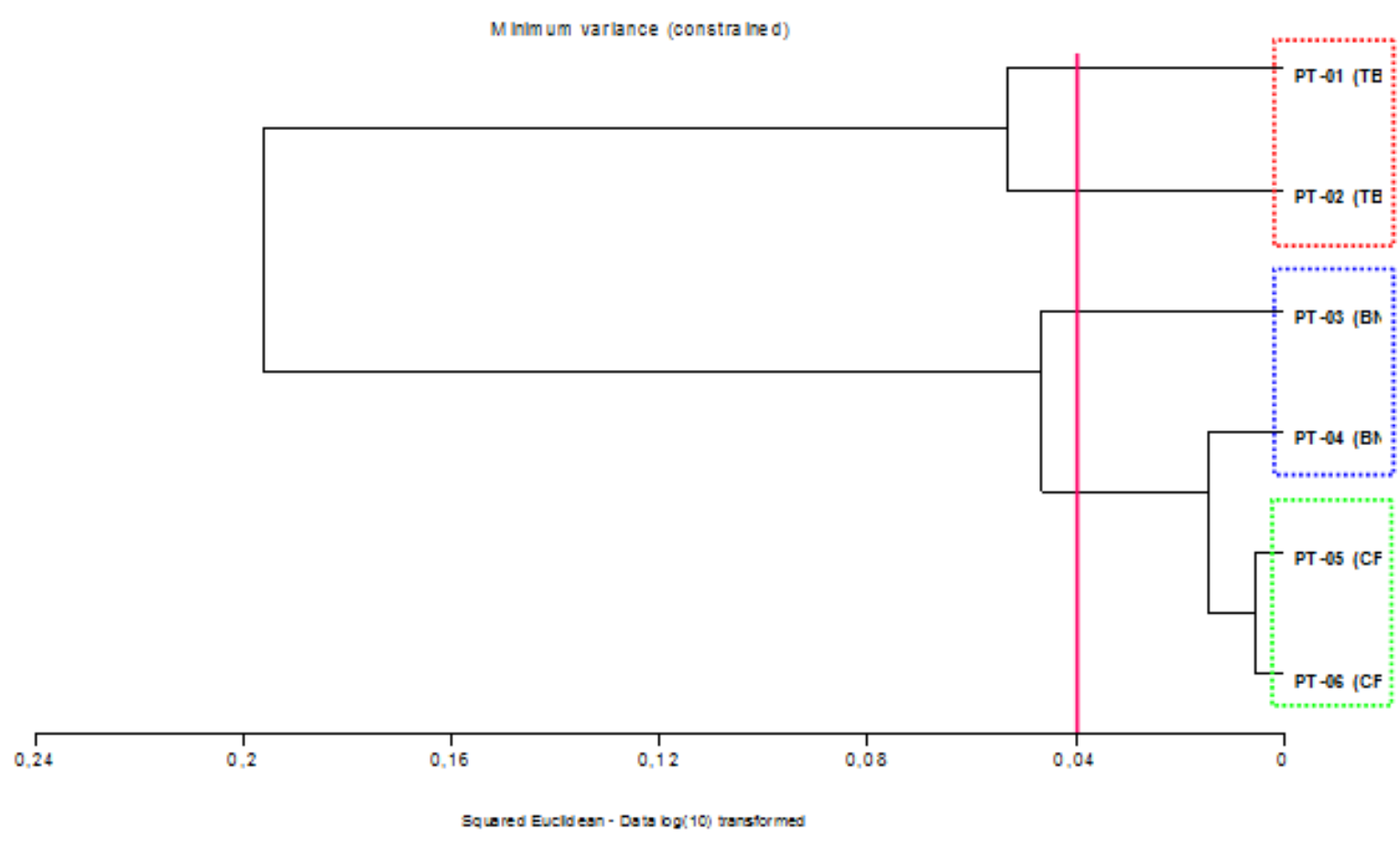

Fonte: Autores (2019).

\section{CONSIDERAÇÕES FINAIS}

A respeito das técnicas estatísticas adotadas, conclui-se que as mesmas demonstraram ser complementares e as interpretações em ACP e agrupamento resultaram em subgrupos de casos, num total de três, com águas do rio Tubarão que destoam 
hidroquimicamente das águas do rio Braço do Norte e, este, faz valer suas características após a confluência.

A partir das interpretações gráficas, e de agrupamento, ficou evidente o condicionamento hidroquímico das águas da bacia, ele é determinado pelo rio Braço do Norte, que funciona como diluidor da carga metálica, alta condutividade e baixo $\mathrm{pH}$ do rio Tubarão.

Por final, se é a natureza química da água a promotora das alterações no processo de sedimentação, como verificado nas bibliografias consultadas, e se esta assinatura hidroquímica é condicionada pelo rio Braço do Norte, como verificado através da interpretação estatística de análises químicas em seis pontos de coleta de água, a hipótese inicial de que a acidez aumentaria as taxas de agradação sedimentar, após o encontro dos rios, é falseada.

\section{REFERÊNCIAS}

BRASIL. Resolução $n^{\circ}$ 420, de 28 de dezembro de 2009. Dispõe sobre critérios e valores orientadores de qualidade do solo quanto à presença de substâncias químicas e estabelece diretrizes para o gerenciamento ambiental de áreas contaminadas por essas substâncias em decorrência de atividades antrópicas. Diário Oficial da União. Brasília, DF, 30 dez. 2009. n. $249, \quad$ p. $\quad 81-84 . \quad$ Disponível em: <http://www.mma.gov.br/port/conama/legiabre.cfm?codlegi=620> Acesso em: $02 \mathrm{mar}$. 2017.

BRASIL. Resolução ${ }^{\circ}$ 454, de 1 de novembro de 2012. Estabelece diretrizes gerais os procedimentos referenciais para o gerenciamento do material a ser dragado em águas sob jurisdição nacional. Diário Oficial da União. Brasília, DF, 8 nov. 2012. n. 216, Seção 1, p. 66-66. Disponível em: 〈http://www.mma.gov.br/port/conama/legiabre.cfm?codlegi=693> Acesso em: 26 jun. 2017.

BROOKFIELD, M. E. The evolution of the great river systems of southern Asia during the Cenozoic India-Asia collision: Rivers draining southwards. Geomorphology, v.22, p. 285312, 1998.

GOTHE, C. A. Diagnóstico Ambiental da Região Carbonífera Catarinense. Curso de Gerência do Meio Ambiente. Florianópolis: Eletrobrás, 03 e 22 de julho de 1988.

FÖRSTNER, U., WITTMAN, G. T. W. Metal pollution in the aquatic environment. 2 ed. Berlin: Springer Verlang, 1983, 486p.

LANDIM, P. M. B. Análise Estatística de Dados Geológicos Multivariados. São Paulo: Oficina de Textos, 2011.

MILIOLI, G. Mineração de Carvão e Desenvolvimento Sustentado no Sul de Santa Catarina. Criciúma: Luana, 1995. 
NETO, J. M.; MOITA, G. C. Uma Introdução à Análise Exploratória de dados Multivariados. Teresina: Química Nova, 1998.

ORFEO, O. Critérios para la clasificacion de barras em grandes rios entrelazados de llanura subtropical. Actas VI Reunion Argentina de Sedimentologia, 1996. pp. 231-236. SANTA CATARINA. Plano Integrado de Recursos Hídricos da Bacia Hidrográfica do rio Tubarão e Complexo Lagunar. Florianópolis: 2002. Disponível em: $<$ http://www.aguas.sc.gov.br/index.php?option=com_k2\&view=item\&layout=item\&id=19 $04 \&$ Itemid $=248 \&$ jsmallfib $=1 \&$ dir $=$ JSROOT/DHRI/Planos + de + Bacias/Plano+de+Bacia $+H$ idrografica+do+Rio+Tubarao+e+Complexo+Lagunar $>$. Acesso em: 07 de mar. 2018. SANTA CATARINA. Lei $\mathbf{n}^{\mathbf{0}} \mathbf{1 0 . 9 4 9}$ de 09 de novembro de 1998. Dispõe sobre a caracterização do Estado em dez Regiões Hidrográficas. Florianópolis: 1998.

SANT ANA, W. O. Qualidade dos recursos hídricos subterrâneos na bacia do rio Urussanga, SC. Dissertação de Mestrado, Programa de Pós-Graduação em Geografia, Florianópolis: UFSC, 2008.

SANTOS, A. L. F.; BORGES, L. O. S., BOAVENTURA, G. R. Indicadores da qualidade dos sedimentos do ribeirão Piancó, Anápolis-GO, e suas implicações ambientais. Scientia Plena. v. 8, n. 10, 2012.

SIRHESC - SISTEMA DE INFORMAÇÕES DE RECURSOS HÍDRICOS DO ESTADO DE SANTA CATARINA. Recursos Hídricos de Santa Catarina. 2017. Disponível em: $\langle$ http://www.aguas.sc.gov.br/base-documental/bacias-hidrograficas-do-estado〉. Acesso em: 14 mar. 2018.

VARELlA, C. A. A. Análise de Componentes Principais. Seropédica: UFRRJ - Pós Graduação em Agronomia, 2008.

WEDEPOHL, K.H. The composition of the continental crust. Geochimica et Cosmochimica Acta. v. 59, n. 7. 1995, p. 1277-1232. 